\title{
Recurrence relations for orthogonal functions
}

\author{
A. Bultheel \\ Department of Computer Science, K.U. Leuven, \\ B-3001 Leuven, Belgium. \\ P. Gonzalez-Vera \\ Facultad de Mathemáticas, Universidad de La Laguna \\ La Laguna, Tenerife, Canary Islands, Spain. \\ E. Hendriksen \\ University of Amsterdam, Department of Mathematics, \\ Plantage Muidergracht 24, 1018 TV Amsterdam, The Netherlands. \\ O. Njåstad \\ Department of Mathematics, University of Trondheim-NTH, \\ N-7034 Trondheim, Norway.
}

\begin{abstract}
Let a sequence $\left\{\alpha_{n}\right\}$ of numbers in the extended complex plane of one of the following four kinds be given: 1) All $\alpha_{n}$ are interior to or exterior to the unit circle. 2) All $\alpha_{n}$ are on the unit circle. 3) All $\alpha_{n}$ are above or below the real line. 4) All $\alpha_{n}$ are on the real line. In each case the sequence $\left\{\alpha_{n}\right\}$ gives rise to a space of rational functions and an orthogonal base in this space with respect to a given functional. Various kinds of recurrence relations satisfied by the elements of this orthogonal base are discussed.
\end{abstract}




\section{Introduction}

In this paper we shall use the following notations: $\boldsymbol{D}=\{z \in C:|z|<1\}, \boldsymbol{E}=\{z \in \boldsymbol{C}$ : $|z|>1\}, \boldsymbol{T}=\{z \in C:|z|=1\}, \boldsymbol{H}_{+}=\{z \in C: \operatorname{Im} z>0\}, \boldsymbol{H}_{-}=\{z \in C: \operatorname{Im} z<$ $0\}, \boldsymbol{R}=\{z \in C: \operatorname{Im} z=0\}$.

The Cayley transform $\tau$ shall here be defined by the formulas

$$
Z=\tau(z)=i \frac{1+z}{1-z}, z=\tau^{-1}(Z)=\frac{Z-i}{Z+i}, \quad z \in D, Z \in \boldsymbol{H}_{+}
$$

The transform $z \rightarrow Z$ maps $\boldsymbol{D}$ onto $\boldsymbol{H}_{+}, \boldsymbol{E}$ onto $\boldsymbol{H}_{-}$and $\boldsymbol{T}$ onto $\boldsymbol{R}$.

The substar conjugate $f_{*}$ of a function $f$ is defined as

$$
f_{*}(z)=\overline{f(1 / \bar{z})}
$$

When $f$ is a rational function, this may be written as

$$
f_{*}(z)=\bar{f}(1 / z)
$$

where the bar denotes complex conjugates of the coefficients. Similarly the subtilde conjugate $f_{\sim}$ is defined by

$$
f_{\sim}(z)=\overline{f(\bar{z})} .
$$

When $f$ is a rational function, this may be written as

$$
f_{\sim}(z)=\bar{f}(z) .
$$

We set $\mathcal{L}_{n *}=\left\{\mathcal{L}_{*}: L \in \mathcal{L}_{n}\right\}, \mathcal{L}_{n \sim}=\left\{L_{\sim}: L \in \mathcal{L}_{n}\right\}$

The setting of this paper will be certain spaces of rational functions with a functional $M$ defined on them. The functional $M$ gives rise to an inner product $<>$ (not necessarily definite) though the formula

$$
<f, g>=M\left(f(z) \cdot g_{*}(z)\right)
$$

or

$$
<f, g>=M\left(f(z) \cdot g_{\sim}(z)\right) .
$$

When $<f, f>\neq 0$ for all $f$ in the space under consideration, we shall call $M$ and $<,>$ quasi-definite, and when $<f, f>>0$ for these $f$ we call $M$ and $<$, > positive definite. 
When $<,>$ is defined by (1.6) we shall talk abaout the circle-disk situation, and when $<,>$ is defined by (1.7) we shall talk about the line-plane situation.

When $\mu$ is a finite (positive) Borel measure with infinite support on $\boldsymbol{T}$ or $\boldsymbol{R}$, positive definite functionals $M$ (an thereby positive definite inner products) are defined through

$$
M(f)=\int_{-\pi}^{\pi} f\left(e^{i \theta}\right) d \mu(\theta)
$$

in the circle-disk situation,

$$
M(f)=\int_{-\infty}^{\infty} f(t) d \mu(t)
$$

in the line-plane situation.

The spaces of rational functions that will be treated arise in connection with NevanlinnaPick interpolation theory. The aim of this paper is to study certain properties of orthogonal bases for these spaces, more specifically recurrence relations connecting elements of these bases.

The theory of orthogonal sequences in these spaces (for the case that all interpolation points are in $\boldsymbol{D}$ ) was initiated by Djrbashian in 1969 (see [14]), and independently by Bultheel, Dewilde and Dym (see $[3,5,13]$ ). For a general introduction to recent work on this theory, see [6]. Other papers discussing these and related spaces are [9,10,25,29]. For earlier work on recurrence relations in these spaces, see $[6,7,8,10,16,17,27,28]$.

When all interpolation points coalesce at one point, the situation is essentially a polynomial situation, and when the interpolation points consist of two points cyclically repeated, the situation is to a large extent a Laurent polynomial situation. In particular when the one point is the origin in the circle-disk situation, orthogonal polynomials on $\boldsymbol{T}$ are obtained, see $[15,21,22,34]$, while when the one point is the point at infinity in the line-plane situation, orthogonal polynomials on $\boldsymbol{R}$ are obtained (see $[1,2,11]$ ). When the two points are the origin and the point at infinity in the line-plane situation, orthogonal Laurent polynomials on $\boldsymbol{R}$ are obtained (see $[18,19,20,24,32])$.

\section{Interpolation in the unit disk}

Let $\left\{\alpha_{n}: n=1,2, \ldots\right\}$ be an arbitrary sequence of (not necessarily distinct) points (interpolation points) in $\boldsymbol{D} \cup \boldsymbol{E}$. We shall always assume that $\alpha_{j} \neq 1 / \overline{\alpha_{k}}$ for $j, k=1,2, \ldots$. Note 
that $1 / \overline{\alpha_{j}} \in \boldsymbol{E}$ if and only if $\alpha_{j} \in \boldsymbol{D}$, hence $\alpha_{j} \neq 1 / \overline{\alpha_{k}}$ is always satisfied if $\alpha_{n} \in \boldsymbol{D}$ for all $n$ or $\alpha_{n} \in \boldsymbol{E}$ for all $n$.

We define the Blaschke factor $\zeta_{n}$ as the function

$$
\zeta_{n}(z)=\frac{\overline{\alpha_{n}}\left(\alpha_{n}-z\right)}{\left|\alpha_{n}\right|\left(1-\overline{\alpha_{n}} z\right)}, n=1,2, \ldots
$$

(By convention we set $\frac{\overline{\alpha_{n}}}{\left|\alpha_{n}\right|}=-1$ if $\alpha_{n}=0$.)

The Blaschke products $B_{n}$ are defined by

$$
B_{0}(z)=1, \quad B_{n}(z)=\prod_{k=1}^{n} \zeta_{k}(z), \quad n=1,2, \ldots
$$

We define the spaces $\mathcal{L}_{n}, \mathcal{L}$ by

$$
\mathcal{L}_{n}=\operatorname{Span}\left\{B_{k}: k=0,1, \ldots, n\right\}, \mathcal{L}=\cup_{n=0}^{\infty} \mathcal{L}_{n}
$$

The functions in $\mathcal{L}_{n}$ are exactly the functions that may be written in the form

$$
L(z)=\frac{p_{n}(z)}{\pi_{n}(z)}
$$

where

$$
\pi_{n}(z)=\prod_{k=1}^{n}\left(1-\overline{\alpha_{k}} z\right), \quad n=1,2, \ldots
$$

and $p_{n} \in \Pi_{n}$ (the space of polynomials of degree at most $n$ ). This follows by partial fraction decomposition. In particular the situation reduces to the polynomial case $\mathcal{L}_{n}=\Pi_{n}$ when $\alpha_{n}=0$ for all $n$.

For $f \in \mathcal{L}_{n}$ we define its superstar conjugate $f^{*}$ by

$$
F^{*}(z)=B_{n}(z) f_{*}(z) \text {. }
$$

Note that this transformation depends on $n$. It must be clear from the context what $n$ is. Also note that $f^{*} \in \mathcal{L}_{n}$ when $f \in \mathcal{L}_{n}$.

Let $M$ be a quasi-definite functional on $\mathcal{L}_{n}+\mathcal{L}_{n *}$, and let $<,>$ be defined by (1.6). Let the sequence $\left\{\Phi_{n}: n=0,1,2, \ldots\right\}$ be obtained by orthogonalization of the sequence $\left\{B_{n}\right\}$ with respect to $<,>$. We shall assume that all $\Phi_{n}$ are monic, which means that the leading coefficient $\kappa_{n}=\kappa_{n}^{(n)}$ in the decomposition

$$
\Phi_{n}(z)=\sum_{k=0}^{n} \kappa_{k}^{(n)} B_{k}(z)
$$


equals one, i.e. $\kappa_{n}^{(n)}=1$.

The following orthogonalitty properties are valid:

$$
\begin{gathered}
<f, \Phi_{n}>=0 \text { for } f \in \mathcal{L}_{n-1} \\
<g, \Phi_{n}^{*}>=0 \text { for } g \in \zeta_{n} \mathcal{L}_{n-1} .
\end{gathered}
$$

Recall that we may write

$$
\Phi_{n}(z)=\frac{P_{n}(z)}{\pi_{n}(z)}, \quad P_{n} \in \Pi_{n} .
$$

By substituting $\alpha_{n}$ for $z$ in the expression for $\Phi_{n}^{*}(z)$ obtained from (2.4), we easily verify that

$$
\Phi_{n}^{*}\left(\alpha_{n}\right)=1
$$

We shall call the index $n$ and the function $\Phi_{n}$ degenerate if $\Phi_{n}^{*}\left(\alpha_{n-1}\right)=0$, nondegenerate otherwise. We shall call the index $n$ and the function $\Phi_{n}$ exceptional if $\Phi_{n}\left(\alpha_{n-1}\right)=0$, non-exceptional otherwise. Note that in the polynomial case $\left(\alpha_{n}=0\right.$ for all $n), \Phi_{n}^{*}\left(\alpha_{n-1}\right)=\Phi_{n}^{*}\left(\alpha_{n}\right)=\Phi_{n}^{*}(0) \neq 0$, so that the degenerate case can not occur.

The following Christoffel-Darboux formula can be shown to be valid (see [6], where only the case $\alpha_{n} \in \boldsymbol{D}$ for all $n$ is explicitely treated) when the inner product $<,>$ is positive definite:

$$
\left[\Phi_{n}^{*}\left(\alpha_{n}\right)\right]^{2}\left[\Phi_{n}^{*}(z) \overline{\Phi_{n}^{*}(w)}-\Phi_{n}(z) \overline{\Phi_{n}(w)}\right]=\left[1-\zeta_{n}(z) \overline{\zeta_{n}(w)}\right] \sum_{k=0}^{n-1}\left[\Phi_{k}^{*}\left(\alpha_{k}\right)\right]^{2} \Phi_{k}(z) \overline{\Phi_{k}(w)}
$$

Proposition 2.1 Assume that the functional $M$ is positive definite, and that $\alpha_{n} \in \boldsymbol{D}$ for all $n$ or $\alpha_{n} \in \boldsymbol{E}$ for all $n$. Then all $\Phi_{n}$ are non-degenerate.

Proof:

By setting $w=z$ in (2.12) we obtain

$$
\left[1-\left|\zeta_{n}(z)\right|^{2}\right]^{-1}\left[\left|\Phi_{n}^{*}(z)\right|^{2}-\left|\Phi_{n}(z)\right|^{2}\right]>0 .
$$

From this it easily follows that $\Phi_{n}^{*}(z) \neq 0$ for $z \in D$ if $\alpha_{n} \in D, \Phi_{n}(z) \neq 0$ for $z \in \boldsymbol{E}$ if $\alpha_{n} \in \boldsymbol{E}$. Thus $\Phi_{n}^{*}\left(\alpha_{n-1}\right) \neq 0$ for all $n$ if $\alpha_{n} \in \boldsymbol{D}$ for all $n$ or $\alpha_{n} \in \boldsymbol{E}$ for all $n$.

Cf. the discussion above of the special case $\alpha_{n}=0$ for all $n$, i.e. the polynomial situation. 


\section{Recurrence in the disk situation}

For the sake of completeness we state a version of the fundamental result on Szegö type recurrence for the orthogonal rational functions.

Theorem 3.1 The sequence $\left\{\Phi_{n}\right\}$ satisfies the following recurrence relation:

$$
\begin{gathered}
\Phi_{n}(z)=\varepsilon_{n} \frac{z-\alpha_{n-1}}{1-\overline{\alpha_{n}} z} \Phi_{n-1}(z)+\delta_{n} \frac{1-\overline{\alpha_{n-1}} z}{1-\overline{\alpha_{n}} z} \Phi_{n-1}^{*}(z), \quad n=1,2, \ldots \\
\Phi_{n}^{*}(z)=\overline{\delta_{n}} \frac{z-\alpha_{n-1}}{1-\overline{\alpha_{n}} z} \Phi_{n-1}(z)+\overline{\varepsilon_{n}} \frac{1-\overline{\alpha_{n-1}} z}{1-\overline{\alpha_{n}} z} \Phi_{n-1}^{*}(z), \quad n=1,2, \ldots \\
\alpha_{0}=0, \quad \Phi_{0}=1, \Phi_{0}^{*}=1 .
\end{gathered}
$$

The coefficients $\delta_{n}, \varepsilon_{n}$ are given by

$$
\begin{gathered}
\delta_{n}=\frac{\left(1-\alpha_{n-1} \overline{\alpha_{n}}\right) \Phi_{n}\left(\alpha_{n-1}\right)}{\left(1-\left|\alpha_{n-1}\right|^{2}\right) \overline{\Phi_{n}^{*}\left(\alpha_{n-1}\right)}} \\
\varepsilon_{n}=-\frac{\overline{\alpha_{n}}\left(1-\overline{\alpha_{n-1}} \alpha_{n}\right) \overline{\Phi_{n}^{*}\left(\alpha_{n-1}\right)}}{\left|\alpha_{n}\right|\left(1-\left|\alpha_{n-1}\right|^{2}\right)} .
\end{gathered}
$$

Proof:

The results follows from [7, Theorem 4.1].

Corrollary 3.2 A function $\Phi_{n}$ (or an index $n$ ) can not be both degenerate and exceptional at the same time.

\section{Proof:}

Follows from (3.1)-(3.4) since $\Phi_{n}(z) \not \equiv 0$.

We note that in the polynomial case we have $\Phi_{n}^{*}(0)=1$ for all $n$, hence $\delta_{n}=\Phi_{n}(0), \varepsilon_{n}=1$ (recall that $\frac{\overline{\alpha_{n}}}{\left(\alpha_{n}\right)}=-1$ in this case), and (3.1)-(3.2) reduces to the classical Szegö formulas

$$
\begin{gathered}
\Phi_{n}(z)=z \Phi_{n-1}(z)+\Phi_{n}(0) \Phi_{n-1}^{*}(z) \\
\Phi_{n}^{*}(z)=\overline{\Phi_{n}(0)} z \Phi_{n-1}(z)+\Phi_{n-1}^{*}(z) .
\end{gathered}
$$

Since in this case $\varepsilon_{n}=1, \Phi_{n}$ can never be degenerate. 
Proposition 3.3 If the functional $M$ is positive definite, and all $\alpha_{n} \in \boldsymbol{D}$ or all $\alpha_{n} \in \boldsymbol{E}$, then $\left|\delta_{n}\right|<\left|\varepsilon_{n}\right|$ for all $n$.

Proof:

We recall that in this situation $\varepsilon_{n} \neq 0$ (see Proposition 2). From (3.4)-(3.5) we get

$$
\frac{\delta_{n}}{\varepsilon_{n}}=-\left(\frac{\overline{\alpha_{n}}}{\left|\alpha_{n}\right|}\right)^{-1} \frac{\Phi_{n}\left(\alpha_{n-1}\right)}{\overline{\Phi_{n}^{*}\left(\alpha_{n-1}\right)}} .
$$

The proof of Proposition 2.1 shows that $\left|\Phi_{n}^{*}\left(\alpha_{m}\right)\right|>\left|\Phi_{n}\left(\alpha_{m}\right)\right|$ for all $m, n$ when all $\alpha_{m} \in \boldsymbol{D}$ and when all $\alpha_{m} \in \boldsymbol{E}$. It follows from (3.8) that $\left|\frac{\delta_{n}}{\varepsilon_{n}}\right|<1$, hence $\left|\delta_{n}\right|<\left|\varepsilon_{n}\right|$.

Theorem 3.4 Assume that $\Phi_{n}$ is non-degenerate. Then the following recurrence relation is satisfied:

$$
\Phi_{n}(z)=\frac{\delta_{n}}{\overline{\varepsilon_{n}}} \Phi_{n}^{*}(z)+\frac{1}{\overline{\varepsilon_{n}}}\left(\left|\varepsilon_{n}\right|^{2}-\left|\delta_{n}\right|^{2}\right) \frac{z-\alpha_{n-1}}{1-\overline{\alpha_{n}} z} \Phi_{n-1}(z), \quad n=1,2, \ldots
$$

In particular, this is the case when the functional $M$ is positive definite and all $\alpha_{n} \in \boldsymbol{D}$ or all $\alpha_{n} \in \boldsymbol{E}$.

Proof:

This formula is obtained by substitution for $\Phi_{n-1}^{*}(z)$ from (3.2) into (3.1), $\varepsilon_{n}$ being different from zero.

We note that in the polynomial case (3.9) reduces to the well-known formula (see e.g. [22])

$$
\Phi_{n}(z)=\delta_{n} \Phi_{n}^{*}(z)+\left(1-\left|\delta_{n}\right|^{2}\right) z \Phi_{n-1}(z) .
$$

We shall call the sequence $\left\{\Phi_{n}\right\}$ non-degenerate if all $\Phi_{n}$ are non-degenerate. We observe that if $\left\{\Phi_{n}\right\}$ is non-degenerate then (3.2) and (3.9) together define a three-term recurrence relation in the sequence $\left\{\Phi_{0}, \Phi_{1}^{*}, \Phi_{1}, \Phi_{2}^{*}, \Phi_{2}, \ldots, \Phi_{n}^{*}, \Phi_{n}, \ldots\right\}$, and thus the elements of this sequence are denominators of a continued fraction. (For basic information on continued fractions, see [23].) We state this property formally in a theorem.

Theorem 3.5 Let $\left\{\Phi_{n}\right\}$ be a non-degenerate sequence, and define

$$
\begin{aligned}
Q_{2 m}(z) & =\Phi_{m}^{*}(z), \quad m=1,2, \ldots \\
Q_{2 m+1}(z) & =\Phi_{m}(z), \quad m=0,1,2, \ldots
\end{aligned}
$$


Then the sequence $\left\{Q_{n}\right\}$ form the denominators of a continued fraction $K_{n=1}^{\infty} \frac{a_{n}(z)}{b_{n}(z)}$, where the elements $a_{n}(z), b_{n}(z)$ are given by

$$
\begin{gathered}
a_{2 m}(z)=\bar{\varepsilon}_{m} \frac{1-\overline{\alpha_{m-1}} z}{1-\overline{\alpha_{m}} z}, \quad a_{2 m+1}(z)=\frac{1}{\overline{\varepsilon_{n}}}\left(\left|\varepsilon_{m}\right|^{2}-\left|\delta_{m}\right|^{2}\right) \frac{z-\alpha_{m-1}}{1-\overline{\alpha_{m}} z}, m=1,2, \ldots \\
b_{2 m}(z)=\overline{\delta_{m}} \frac{z-\alpha_{m-1}}{1-\overline{\alpha_{m}} z}, \quad b_{2 m+1}(z)=\frac{\delta_{m}}{\overline{\varepsilon_{m}}}, m=1,2, \ldots
\end{gathered}
$$

The recurrence relation satisfied by the denominators $\left\{Q_{n}\right\}$ are thus

$$
\begin{gathered}
Q_{2 m}(z)=\overline{\delta_{m}} \frac{z-\alpha_{m-1}}{1-\overline{\alpha_{m}} z} Q_{2 m-1}(z)+\overline{\varepsilon_{m}} \frac{1-\overline{\alpha_{m-1}} z}{1-\overline{\alpha_{m}} z} Q_{2 m-2}(z),, \quad m=1,2, \ldots \\
Q_{2 m+1}(z)=\frac{\delta_{m}}{\bar{\varepsilon}_{m}} Q_{2 m}(z)+\frac{1}{\overline{\varepsilon_{m}}}\left(\left|\varepsilon_{m}\right|^{2}-\left|\delta_{m}\right|^{2}\right) \frac{z-\alpha_{m-1}}{1-\overline{\alpha_{m}} z} Q_{2 m-1}(z), \quad m=1,2, \ldots \\
\alpha_{0}=0, Q_{0}=1, Q_{1}=1 .
\end{gathered}
$$

In particular the above results hold when the functional $M$ is positive definite and all $\alpha_{n} \in \boldsymbol{D}$ or all $\alpha_{n} \in \boldsymbol{E}$.

In the polynomial case (all $\alpha_{n}=0$ ) the continued fractions above reduce to the well-known PC-fractions (Perron-Carathéodory fractions), see [21,22]. The PC-fractions are closely related to the Carathéodory Coefficient Problem. The continued fractions described in Theorem 3.5 shall be called NP-fractions (Nevanlinna-Pick fractions). These continued fractions are related to the Nevanlinna-Pick Interpolation Problem (cf. [1,26,33]) in a way that directly generalizes the relationship between PC-fractions and the Carathéodory Coefficient Problem. For connections between the Nevanlinna-Pick Problem and general system theory, see e.g. [12].

We shall call the sequence $\left\{\Phi_{n}\right\}$ non-exceptional if all $\Phi_{n}$ are non-exceptional.

Theorem 3.6 Assume that $\Phi_{n-1}$ is non-exceptional. Then the following recurrence relation is valid:

$$
\begin{aligned}
\Phi_{n}(z) & =\frac{1}{1-\overline{\alpha_{n}} z}\left[\varepsilon_{n}\left(z-\alpha_{n-1}\right)+\overline{\varepsilon_{n}} \frac{\delta_{n}}{\delta_{n-1}}\left(1-\overline{\alpha_{n-1}} z\right)\right] \Phi_{n-1}(z) \\
& -\frac{\delta_{n}}{\delta_{n-1}} \frac{\left(z-\alpha_{n-2}\right)}{\left(1-\overline{\alpha_{n}} z\right)}\left[\left|\varepsilon_{n-1}\right|^{2}-\left|\delta_{n-1}\right|^{2}\right] \Phi_{n-2}(z) n=2,3, \ldots
\end{aligned}
$$




$$
\begin{gathered}
\Phi_{n}^{*}(z)=\frac{1}{1-\overline{\alpha_{n}} z}\left[\overline{\varepsilon_{n}}\left(1-\overline{\alpha_{n-1}} z\right)+\frac{\varepsilon_{n-1} \overline{\delta_{n}}}{\overline{\delta_{n-1}}}\left(z-\alpha_{n-1}\right)\right] \Phi_{n-1}^{*}(z) \\
-\frac{\overline{\delta_{n}}}{\overline{\delta_{n-1}}}\left[\left|\varepsilon_{n-1}\right|^{2}-\left|\delta_{n-1}\right|^{2}\right] \frac{\left(z-\alpha_{n-1}\right)\left(1-\overline{\alpha_{n-2}} z\right)}{\left(1-\overline{\alpha_{n}} z\right)\left(1-\overline{\alpha_{n-1}} z\right)} \Phi_{n-2}^{*}(z) n=2,3, \ldots \\
\Phi_{0}=1, \quad \Phi_{0}^{*}=1, \quad \Phi_{1}(z)=\frac{\delta_{1}+\varepsilon_{1} z}{1-\bar{\alpha}_{1} z_{1}} \Phi_{1}^{*}(z)=\frac{\overline{\varepsilon_{1}}+\overline{\delta_{1}} z}{1-\overline{\alpha_{1}} z} .
\end{gathered}
$$

Proof:

Immediately from (3.1)-(3.2) with $n$ replaced by $n-1$ we obtain

$$
\begin{gathered}
\Phi_{n-2}^{*}(z)=\frac{1}{\delta_{n-1}} \cdot \frac{1-\overline{\alpha_{n-1}} z}{1-\overline{\alpha_{n-2}} z} \Phi_{n-1}(z)-\frac{\varepsilon_{n-1}}{\delta_{n-1}} \frac{z-\alpha_{n-2}}{1-\overline{\alpha_{n-2}} z} \Phi_{n-2}(z) \\
\Phi_{n-2}(z)=\frac{1}{\overline{\delta_{n-1}}} \cdot \frac{1-\overline{\alpha_{n-1}} z}{z-\alpha_{n-2}} \Phi_{n-1}^{*}(z)-\frac{\overline{\varepsilon_{n-1}}}{\overline{\delta_{n-1}}} \cdot \frac{1-\overline{\alpha_{n-2}} z}{z-\alpha_{n-2}} \Phi_{n-2}^{*}(z) .
\end{gathered}
$$

By substituting from (3.21) for $\Phi_{n-1}^{*}$ and $\Phi_{n-2}^{*}$ in (3.2) with $n$ replaced by $n-1$, we obtain (3.18). Similarly by substituting from (3.22) for $\Phi_{n-1}$ and $\Phi_{n-2}$ in (3.1) with $n$ replaced by $n-1$, we obtain (3.19).

Theorem 3.7 Let $\left\{\Phi_{n}\right\}$ be a non-exceptional sequence. Then $\left\{\Phi_{n}\right\}$ form the denominators of a continued fraction $K_{n=1}^{\infty} \frac{a_{n}(z)}{b_{n}(z)}$, where the elements $a_{n}(z), b_{n}(z)$ are given by

$$
\begin{gathered}
a_{n}(z)=\frac{\delta_{n}}{\delta_{n-1}} \frac{z-\alpha_{n-2}}{1-\overline{\alpha_{n}} z}\left(\left|\delta_{n-1}\right|^{2}-\left|\varepsilon_{n-1}\right|^{2}\right) \\
b_{n}(z)=\frac{1}{1-\overline{\alpha_{n}} z}\left[\varepsilon_{n}\left(z-\alpha_{n-1}\right)+\overline{\varepsilon_{n}} \frac{\delta_{n}}{\delta_{n-1}}\left(1-\overline{\alpha_{n-1}} z\right)\right], \quad n=2,3, \ldots,
\end{gathered}
$$

and $\left\{\Phi_{n}^{*}\right\}$ form the denominators of a continued fraction $K_{n=1}^{\infty} \frac{c_{n}(z)}{d_{n}(z)}$, where the elements $c_{n}(z), d_{n}(z)$ are given by

$$
\begin{gathered}
c_{n}(z)=\frac{\overline{\delta_{n}}}{\overline{\delta_{n-1}}}\left[\left|\delta_{n-1}\right|^{2}-\left|\varepsilon_{n-1}\right|^{2}\right] \frac{\left(z-\alpha_{n-1}\right)\left(1-\overline{\alpha_{n-2}} z\right)}{\left(1-\overline{\alpha_{n}} z\right)\left(1-\overline{\alpha_{n-1}} z\right)}, \\
d_{n}(z)=\frac{1}{1-\overline{\alpha_{n}} z}\left[\overline{\varepsilon_{n}}\left(1-\overline{\alpha_{n-1}} z\right)+\frac{\varepsilon_{n-1} \overline{\delta_{n}}}{\overline{\delta_{n-1}}}\left(z-\alpha_{n-1}\right)\right], \quad n=2,3, \ldots .
\end{gathered}
$$

The recurrence relations have the form (3.18)-(3.20). 


\section{Proof:}

Immediate from Theorem 3.6.

In the polynomial case $\left(\alpha_{n}=0\right.$ for all $\left.n\right)$, the formulas (3.18)-(3.19) reduce to the formulas

$$
\begin{gathered}
\Phi_{n}(z)=\left(z+\frac{\delta_{n}}{\delta_{n-1}}\right) \Phi_{n-1}(z)-\left(1-\left|\delta_{n-1}\right|^{2}\right) \frac{\delta_{n}}{\delta_{n-1}} z \Phi_{n-2}(z) \\
\Phi_{n}^{*}(z)=\left(1+\frac{\overline{\delta_{n}}}{\overline{\delta_{n-1}}} z\right) \Phi_{n-1}^{*}(z)-\frac{\overline{\delta_{n}}}{\overline{\delta_{n-1}}}\left(1-\mid \delta_{n-1} 1^{2}\right) z \Phi_{n-2}^{*}(z) .
\end{gathered}
$$

These are $M$-fractions and general T-fractions, respectively. (See [23].) Continued fractions of the form (3.18) or (3.19) are instances of MP-fractions (Multipoint Padé continued fractions), see Section 5 (cf. $[17,30]$ ). They bear the same relationship to multipoint Padé approximation as general T-fractions and $\mathrm{M}$-fractions to two-point Padé approximation.

For a short treatment of continued fractions obtained from the basic recurrence formulas (3.1)-(3.2), see also [6], with reference to [4].

\section{Interpolation on the unit circle}

Let $\left\{\alpha_{n}: n=1,2, \ldots\right\}$ be an arbitrary sequence of (not necessarily distinct) points on $\boldsymbol{T}$. Note that $1 / \overline{\alpha_{n}}=\alpha_{n}$. We introduce functions $\omega_{n}$ by

$$
\omega_{0}=1, \omega_{n}(z)=\prod_{k=1}^{n}\left(z-\alpha_{k}\right), \quad n=1,2, \ldots,
$$

and define the spaces $\mathcal{L}_{n}$ and $\mathcal{L}$ by

$$
\mathcal{L}_{n}=\operatorname{Span}\left\{\frac{1}{\omega_{0}}, \frac{1}{\omega_{1}}, \ldots, \frac{1}{\omega_{n}}\right\}, \mathcal{L}=\cup_{n=0}^{\infty} \mathcal{L}_{n} .
$$

We may also write

$$
\mathcal{L}_{n}=\operatorname{Span}\left\{\frac{z^{m_{0}}}{\omega_{0}}, \frac{z^{m_{1}}}{\omega_{1}(z)}, \ldots, \frac{z^{m_{n}}}{\omega_{n}(z)}\right\}, 0 \leq m_{k} \leq k
$$

and

$$
\mathcal{L}_{n}=\operatorname{Span}\left\{\sigma_{0}, \sigma_{1}, \ldots, \sigma_{n}\right\},
$$

where

$$
\sigma_{0}=1, \quad \sigma_{n}=\frac{i^{n}(1+z)^{n} \prod_{k=1}^{n}\left(1+\alpha_{k}\right)}{\omega_{n}(z)}, n=1,2, \ldots .
$$


(We have for convenience assumed that $z=-1$ is not among the points $\alpha_{n}$. If $\alpha_{n}=-1$ for some $n$, a slightly different definition can be used.)

We observe that $\sigma_{n *}=\sigma_{n}$, and $\mathcal{L}_{n *}=\left\{\boldsymbol{L}_{*}: L \in \mathcal{L}_{n}\right\}=\mathcal{L}_{n}$.

The functions in $\mathcal{L}_{n}$ are exactly the functions that may be written in the form

$$
L(z)=\frac{p_{n}(z)}{\omega_{n}(z)}, p_{n} \in \Pi_{n} .
$$

(This follows by partial fraction decomposition).

We shall have occasion to work also with the spaces $\mathcal{L}_{n} \cdot \mathcal{L}_{n}$ and $\mathcal{L} \cdot \mathcal{L}$. Note that $\mathcal{L}_{n} \cdot \mathcal{L}_{n} \subset \mathcal{L}_{n}, \mathcal{L} \cdot \mathcal{L} \subset \mathcal{L}$, since $1 \in \mathcal{L}_{n}$ for all $n$

Let $M$ be a linear functional on $\mathcal{L} \cdot \mathcal{L}$, and assume that $M$ has real values for all functions in $\mathcal{L}$ that are real on $\boldsymbol{T}$. As before we define the inner product $<,>$ by (1.6), and assume that this inner product is quasidefinite.

Let $\left\{\Phi_{n}\right\}$ be the monic orthogonal system obtained by the Gram-Schmidt process from the sequence $\left\{\sigma_{n}\right\}$ (or equivalently from the sequence $\left\{\frac{1}{\omega_{n}}\right\}$ ). That $\Phi_{n}$ is monic means that the coefficient of $\sigma_{n}$ in the expansion of $\Phi_{n}$ is one. Thus

$$
\Phi_{n}(z)=\sum_{m=0}^{n} b_{m}^{(n)} \sigma_{m}(z), \quad b_{n}^{(n)}=1, \quad b_{m}^{(n)} \in R .
$$

We may write

$$
\Phi_{n}(z)=\frac{P_{n}(z)}{\omega_{n}(z)}, P_{n} \in \Pi_{n}
$$

We easily verify that

$$
\Phi_{n *}(z)=\Phi_{n}(z), \quad n=0,1,2, \ldots
$$

Note that $P_{n}\left(\alpha_{n}\right) \neq 0$ for all $n$. We shall call the function $\Phi_{n}$ and the index $n$ singular if $P_{n}\left(\alpha_{n-1}\right)=0$. (Note that the properties of being degenerate and of being exceptional coincide in the circle situation, because of (4.9), and we use the word singular for these coinciding phenomena in this case.) We shall call $\Phi_{n}$ and $n$ regular if $P_{n}\left(\alpha_{n-1}\right) \neq 0$.

\section{Recurrence in the circle situation}

We shall in this section state without proof a general result on three-term recursion in the circle situation, and briefly discuss two special cases with one interpolation point repeated and with two interpolation points cyclically repeated. 
Theorem 5.1 Assume that the system $\left\{\Phi_{n}\right\}$ is regular. Then a recurrence relation of the following form holds:

$$
\Phi_{n}(z)=\left(\frac{A_{n}}{z-\alpha_{n}}+B_{n} \frac{z-\alpha_{n-2}}{z-\alpha_{n}}\right) \Phi_{n-1}(z)+C_{n} \frac{z-\alpha_{n-2}}{z-\alpha_{n}} \Phi_{n-2}(z), \quad n=2,3, \ldots
$$

with the initial condition

$$
\alpha_{0}=0 .
$$

The constants $A_{n}, B_{n}, C_{n}$ satisfy the inequalities

$$
\begin{gathered}
A_{n}+B_{n}\left(\alpha_{n-1}-\alpha_{n-2}\right) \neq 0, \quad n=2,3, \ldots \\
C_{n} \neq 0, \quad n=2,3, \ldots .
\end{gathered}
$$

Proof:

See [10]. Cf. also [16], which treats the cyclic situation in the line case (discussed in Section $6)$.

It follows that the orthogonal functions $\left\{\Phi_{n}\right\}$ are denominators of a continued fraction $K_{n=1}^{\infty} \frac{a_{n}(z)}{b_{n}(z)}$, with elements

$$
\begin{gathered}
a_{n}(z)=\frac{C_{n}\left(z-\alpha_{n-2}\right)}{z-\alpha_{n}} \\
b_{n}(z)=\left(\frac{A_{n}}{z-\alpha_{n}}+B_{n} \frac{z-\alpha_{n-2}}{z-\alpha_{n}}\right) .
\end{gathered}
$$

Continued fractions of this form have been called MP-fractions (Multipoint Padé continued fractions). See [17] for further discussion in the cyclic case. Note that if $\alpha_{n}=1$ for all $n$, then $\Phi_{n}$ is of the form

$$
\Phi_{n}(z)=\sum_{m=1}^{n} \frac{\beta_{m}}{(z-1)^{m}}
$$

Corrollary 5.2 Let $\alpha_{n}=1$ for $n=1,2, \ldots$. Then the sequence $\left\{\Phi_{n}\right\}$ satisfies a recurrence relation of the following form:

$$
\begin{gathered}
\Phi_{n}(z)=\left(\frac{A_{n}}{z-1}+B_{n}\right) \Phi_{n-1}(z)+C_{n} \Phi_{n-2}(z), n=2,3, \ldots \\
A_{n} \neq 0 \text { for } n=2,3, \ldots \\
C_{n} \neq 0 \text { for } n=2,3, \ldots
\end{gathered}
$$


Proof:

Follows directly from Theorem 5.1, when we recall that $\Phi_{n}$ is always regular in this case. (The case $n=2$ must be handled separately.)

For technical reasons we introduced the assumption $\alpha_{n} \neq-1$ for all $n$. It can be shown that a recurrence relation of the form (5.1) is valid also in the case that $\alpha_{n}=-1$ for some $n$.

Corrollary 5.3 Let $\alpha_{2 m}=1, m=1,2, \ldots, \alpha_{2 m+1}=-1, m=0,1,2, \ldots$. Assume that the sequence $\left\{\Phi_{n}\right\}$ is regular. Then $\left\{\Phi_{n}\right\}$ satisfies a recurrence relation of the following form:

$$
\begin{gathered}
\Phi_{2 m}(z)=\left(\frac{A_{2 m}}{z-1}+B_{2 m}\right) \Phi_{2 m-1}(z)+C_{2 m} \Phi_{2 m-2}(z) m=1,2, \ldots \\
\Phi_{2 m+1}(z)=\left(\frac{A_{2 m+1}}{z+1}+B_{2 m+1}\right) \Phi_{2 m}(z)+C_{2 m+1} \Phi_{2 m-1}(z) m=1,2 . .
\end{gathered}
$$

Proof:

Follows directly from Theorem 5.1.

\section{Orthogonality and recurrence in the plane situa- tion}

Let $\left\{A_{n}\right\}$ be an arbitrary sequence of (not necessarily distinct) points in $\boldsymbol{H}_{+} \cup \boldsymbol{H}_{-}$, and assume that $A_{j} \neq \overline{A_{k}}$ for all $j, k$. Set

$$
\alpha_{n}=\frac{A_{n}-i}{A_{n}+i}
$$

cf. (1.1).

Then $\alpha_{n} \in \boldsymbol{D} \cup \boldsymbol{E}$, and $\alpha_{j} \neq 1 / \overline{\alpha_{k}}$ for $j \neq k$. To the functions $\zeta_{n}(z)$ correspond the functions $\Gamma_{n}(Z)$ defined by

$$
\Gamma_{n}(Z)=\frac{z-A_{n}}{z-\overline{A_{n}}}
$$

Define $\Delta_{n}$ by

$$
\Delta_{0}=1, \quad \Delta_{n}(Z)=\prod_{k=1}^{n} \Gamma_{k}(Z), \quad n=1,2, \ldots,
$$


and set

$$
\mathcal{M}_{n}=\operatorname{Span}\left\{\Delta_{0}, \Delta_{1}, \ldots, \Delta_{n}\right\}, \mathcal{M}=\cup_{n=0}^{\infty} \mathcal{M}_{n}
$$

We set $\mathcal{M}_{n \sim}=\left\{M_{\sim}: M \in \mathcal{M}_{n}\right\}, \mathcal{M}_{\sim}=\cup_{n=0}^{\infty} \mathcal{M}_{m \sim}$. Let $M$ be a linear functional on $\mathcal{M}+\mathcal{M}_{\sim}$, and let $<,>$ be defined by (1.7). The functions $F_{n}$ defined by

$$
F_{n}(Z)=\Phi_{n}(z)=\Phi_{n}\left(\frac{Z-i}{Z+i}\right)
$$

are orthogonal functions corresponding to the bases $\left\{\mathcal{M}_{n}\right\}$. (Here $\left\{\Phi_{n}\right\}$ are orthogonal with respect to the inner product (1.6) corresponding to the bases $\left\{\mathcal{L}_{n}\right\}$.)

We define

$$
f_{n}^{\sim}(Z)=\Delta_{n}(Z) f_{n \sim}(Z)
$$

when $f_{n} \in \mathcal{M}_{n}$, and note that $f_{n}^{\sim} \in \mathcal{M}_{n}$.

We further observe that

$$
F_{n}^{\sim}(Z)=\Phi_{n}^{*}(z), \quad z=\frac{Z-i}{Z+i}
$$

We call $F_{n}$ degenerate if $F_{n}^{\sim}\left(A_{n-1}\right)=0$, exceptional if $F_{n}\left(A_{n-1}\right)=0$.

Note that if $Z=\tau(z), \quad A=\tau(\alpha)(\mathrm{cf} .(1.1))$, then

$$
\begin{aligned}
z-\alpha & =\frac{2 i(Z-A)}{(Z+i)(A+i)} \\
1-\bar{\alpha} z & =\frac{2 i(Z-\bar{A})}{(i-\bar{A})(Z+i)} .
\end{aligned}
$$

Theorem 6.1 The sequence $\left\{F_{n}\right\}$ satisfies a recurrence relation of the following form:

$$
\begin{gathered}
F_{n}(Z)=E_{n} \frac{Z-A_{n-1}}{Z-\overline{A_{n}}} F_{n-1}(Z)+D_{n} \frac{Z-\overline{A_{n-1}}}{Z-\overline{A_{n}}} F_{n-1}^{\sim}(Z) \\
F_{n}^{\sim}(Z)=\overline{D_{n}} \frac{Z-A_{n-1}}{Z-\overline{A_{n}}} F_{n-1}(Z)+\overline{E_{n}} \frac{Z-\overline{A_{n-1}}}{Z-\overline{A_{n}}} F_{n-1}^{\sim}(Z), \quad n=1,2, \ldots \\
A_{0}=i, \quad F_{0}=1, \quad F_{0}^{\sim}=1 .
\end{gathered}
$$


Proof:

Follows from Theorem 3.1 by the substitutions (6.7), (6.8), (6.9).

Theorem 6.2 Assume that $F_{n}$ is non-degenerate. Then $F_{n}$ satisfies a recurrence relation of the following form:

$$
F_{n}(Z)=\frac{\overline{D_{n}}}{\overline{E_{n}}} F_{n}^{\sim}(Z)+\frac{1}{\overline{E_{n}}}\left(\left|E_{n}\right|^{2}-\left|D_{n}\right|^{2}\right) \frac{Z-A_{n}}{Z-\overline{A_{n}}} F_{n-1}(Z)
$$

In particular this is the case if the functional $M$ is positive definite and all $A_{n} \in \boldsymbol{H}_{+}$or all $A_{n} \in \boldsymbol{H}_{-}$.

\section{Proof:}

Follows as above from Theorem 3.4.

It follows by combining $(6.11)$ and $(6.13)$ that if the sequence $\left\{F_{n}\right\}$ is non-degenerate, then the elements of the sequence $\left\{F_{0}, F_{1}^{\sim}, F_{1}, F_{2}^{\sim}, F_{2}, \ldots, F_{n}^{\sim}, F_{n}, \ldots\right\}$ are the denominators of a continued fraction, just as in the circle case. We shall call also these continued fractions NP-fractions.

Theorem 6.3 Assume that $\left\{F_{n}\right\}$ is non-exceptional. Then $\left\{F_{n}\right\}$ and $\left\{F_{n}^{\sim}\right\}$ satisfy recurrence relations of the following form:

$$
\begin{gathered}
F_{n}(Z)=\left[\frac{E_{n}\left(Z-A_{n-1}\right)}{Z-\overline{A_{n}}}+\frac{\overline{E_{n}} D_{n}\left(Z-\overline{A_{n-1}}\right)}{D_{n-1}\left(Z-\overline{A_{n}}\right)}\right] F_{n-1}(Z) \\
-\frac{D_{n}\left(Z-A_{n-2}\right)}{D_{n-1}\left(Z-\overline{A_{n}}\right)}\left[\left|E_{n-1}\right|^{2}-\left|D_{n-1}\right|^{2}\right] F_{n-2}(Z), \quad n=2,3, \ldots \\
F_{n}^{\sim}(Z)=\left[\frac{\overline{E_{n}}\left(Z-\overline{A_{n-1}}\right)}{Z-\overline{A_{n}}}+\frac{E_{n-1}\left(Z-A_{n-1}\right)}{\overline{D_{n-1}}\left(Z-\overline{A_{n}}\right)}\right] F_{n-1}^{\sim}(Z) \\
-\frac{\overline{D_{n}}}{\overline{D_{n-1}}}\left[\left|E_{n-1}\right|^{2}-\left|D_{n-1}\right|^{2}\right] \frac{\left(Z-A_{n-1}\right)\left(Z-\overline{A_{n-2}}\right)}{\left(Z-\overline{A_{n}}\right)\left(Z-\overline{A_{n-1}}\right)} F_{n-2}^{\sim}(Z), \quad n=2,3, \ldots \\
F_{0}=1, \quad F_{0}^{\sim}=1, \quad F_{1}(Z)=\frac{D_{1}+E_{1} Z}{Z-\overline{A_{1}}}, F_{1}^{\sim}=\frac{\overline{E_{1}}+\overline{D_{1}} Z}{Z-\overline{A_{1}}} .
\end{gathered}
$$

Proof:

Follows as above from Theorem 3.6. 
It follows that if the sequence $\left\{F_{n}\right\}$ is non-exceptional, then the elements of the sequences $\left\{F_{n}\right\}$ and $\left\{F_{n}^{\sim}\right\}$ are the denominators of two continued fractions. These are again instances of MP-fractions. (See Section 4,5.)

In the special case $A_{n}=i$ for all $n$ (corresponding to the polynomial case in the circle situation) these formulas reduce to

$$
\begin{gathered}
F_{n}(Z)=\left[E_{n} \frac{Z-i}{Z+i}+\frac{\overline{E_{n}} D_{n}}{D_{n-1}}\right] F_{n-1}(Z)-\frac{D_{n}}{D_{n-1}}\left[\left|E_{n-1}\right|^{2}-\left|D_{n-1}\right|^{2}\right] \frac{Z-i}{Z+i} F_{n-2}(Z) \\
F_{n}^{\sim}(Z)=\left[\overline{E_{n}}+\frac{\overline{E_{n-1}}}{\overline{D_{n-1}}} \frac{(Z-i)}{(Z+i)}\right] F_{n-1}^{\sim}(Z)-\frac{\overline{E_{n} D_{n}}}{\overline{D_{n-1}}}\left[\left|E_{n-1}\right|^{2}-\left|D_{n-1}\right|^{2}\right] \frac{Z-i}{Z+i} F_{n-2}^{\sim}(Z) .
\end{gathered}
$$

\section{Orthogonality and recurrence in the line situation}

Let $\left\{A_{n}\right\}$ be an arbitrary sequence of (not necessarily distinct) points on $\boldsymbol{R}$. Define the points $\alpha_{n}$ as in Section 6:

$$
\alpha_{n}=\frac{A_{n}-i}{A_{n}+i}
$$

Then $\alpha_{n} \in \boldsymbol{T}$. To the functions $\omega_{n}(z)$ defined in (4.1) correspond the functions $\Omega_{n}(Z)$ defined by

$$
\Omega_{0}=1, \quad \Omega_{n}=\prod_{k=1}^{n}\left(Z-A_{k}\right), \quad n=1,2, \ldots .
$$

We set

$$
\mathcal{M}_{n}=\operatorname{Span}\left\{\frac{1}{\Omega_{0}}, \frac{1}{\Omega_{n}}, \ldots, \frac{1}{\Omega_{n}}\right\}, \mathcal{M}=\cup_{n=0}^{\infty} \cdot \mathcal{U}_{n} .
$$

Let $\mathcal{L}_{n}, \mathcal{L}$ be the spaces defined by (4.2).

Let $M$ be a functional on $\mathcal{M} \cdot \mathcal{M}$ with $<,>$ defined by (1.7). The functions $F_{n}$ defined by

$$
F_{n}(Z)=\Phi_{n}(z)=\Phi_{n}\left(\frac{Z-i}{Z+i}\right)
$$

are orthogonal functions corresponding to the bases $\left\{\mathcal{M}_{n}\right\}$. (Here $\left\{\Phi_{n}\right\}$ are orthogonal with respect to the inner product (1.6), corresponding to the bases $\left\{\mathcal{L}_{n}\right\}$.)

We may write $F_{n}$ in the form

$$
F_{n}(Z)=\frac{P_{n}(Z)}{\Omega_{n}(Z)}, P_{n} \in \Pi_{n} .
$$


We shall call $F_{n}$ singular if $P_{n}\left(A_{n-1}\right)=0$. (Here as in the circle situation, the properties of being degenerate and of being exceptional coincide, and we use the word singular for these coinciding phenomena in this case.) We call $F_{n}$ regular if $P_{n}\left(A_{n-1}\right) \neq 0$.

Theorem 7.1 Assume that the system $\left\{F_{n}\right\}$ is regular. Then a recurrence relation of the following form holds:

$$
F_{n}(Z)=\left[\frac{a_{n}}{Z-A_{n}}+\frac{b_{n}\left(Z-A_{n-2}\right)}{Z-A_{n}}\right] F_{n-1}(Z)+C_{n} \frac{Z-A_{n-2}}{Z-A_{n}} F_{n-2}(Z), \quad n=2,3, \ldots,
$$

with initial condition

$$
A_{0}=i
$$

Proof:

Follows from Theorem 5.1 by the substitutions (1.1) and (7.4).

Let $A_{n}=\infty$ for all $n$. By (1.1) this corresponds to $\alpha_{n}=1$ for all $n$. In this case $\Phi_{n}(z)$ are of the form

$$
\Phi_{n}(z)=\sum_{k=0}^{n} \frac{\beta_{k}}{(z-1)^{k}},
$$

see (5.7). By substituting for $z$ from (1.1) we get

$$
F_{n}(Z)=\sum_{k=0}^{n} \gamma_{k} Z^{k}
$$

which is a polynomial. Thus $A_{n}=\infty$ for all $n$ represents the polynomial situation.

Corrollary 7.2 Let $A_{n}=\infty$ for all $n$. Then the sequence $\left\{F_{n}\right\}$ satisfies a recurrence relation of the form

$$
F_{n}(Z)=\left(a_{n} Z+b_{n}\right) F_{n-1}(Z)+C_{n} F_{n-2}(Z), \quad n=2,3,4 \ldots
$$

Proof:

Follows from Corollary 5.2 by the substitutions (1.1) and (7.4).

Let $A_{2 m}=\infty, m=1,2, \ldots, A_{2 m+1}=0, m=0,1,2, \ldots$. Then the orthogonal functions $\Phi_{n}(z)$ are of the form

$$
\Phi_{2 m}(z)=\sum_{k=0}^{m} \frac{\beta_{k}}{(z-1)^{k}}+\sum_{k=0}^{m} \frac{\gamma_{k}}{(z+1)^{k}},
$$




$$
\Phi_{2 m+1}(z)=\sum_{k=0}^{m} \frac{\beta_{k}}{(z-1)^{k}}+\sum_{k=0}^{m+1} \frac{\gamma_{k}}{(z+1)^{k}} .
$$

By substituting in (7.11)-(7.12) from (1.1) and (7.4) we get

$$
\begin{gathered}
F_{2 m}(Z)=\sum_{k=-m}^{m} \kappa_{k} Z^{k} \\
F_{2 m+1}(Z)=\sum_{k=-(m+1)}^{m} \kappa_{k} Z^{k} .
\end{gathered}
$$

These functions are orthogonal Laurent polynomials.

Corrollary 7.3 Let $A_{2 m}=\infty$ for $m=1,2, \ldots, A_{2 m+1}=0$ for $m=0,1,2, \ldots$. Assume that the sequence $\left\{F_{n}\right\}$ is regular. Then $\left\{F_{n}\right\}$ satisfies a recurrence relation of the following form:

$$
\begin{gathered}
F_{2 m}(Z)=\left(a_{2 m} Z+b_{2 m}\right)\left(F_{2 m-1}(Z)+c_{2 m} F_{2 m-2}(Z), m=1,2,3, \ldots\right. \\
F_{2 m+1}(Z)=\left(\frac{a_{2 m+1}}{Z}+b_{2 m+1}\right) F_{2 m}(Z)+c_{2 m+1} F_{2 m-1}(Z), \quad m=0,1,2, \ldots .
\end{gathered}
$$

Proof:

Follows from Corrollary 5.3 by the substitutions (1.1) and (7.4).

The above recurrence relations are essentially the well-known recurrence relations for regular orthogonal Laurent polynomials. (See [20,32]. Cf. also [31].) 


\section{REFERENCES}

1. N.I. Akhiezer, The classical moment problem and some related questions in analysis, (Hafner, New York, 1965).

2. C. Brezinski, Padé-Type Approximants and Orthogonal Polynomials, Birkhäuser, Basel/Boston/Stuttgart, 1980.

3. A. Bultheel, Orthogonal matrix functions related to the multivariable NevanlinnaPick problem, Tijdschr. Belgisch Wisk. Genootenshap, Ser. B. 32 (2) (1980) 149-170.

4. A. Butheel. Laurent series and their Padé approximations, volume 27 of Operator Theory: advances and applications. Birkhäuser Verlag, Basel, 1987.

5. A. Bultheel and P. Dewilde, Orthogonal functions related to the Nevanlinna-Pick problem, Mathematical Theory of Networks and Systems, Proceedings MTNS Conference, Delft, The Nerherlands (Ed. P. Dewilde) Western Periodicals, North Hollywood, 1979, 207-211.

6. A. Bultheel, P. Gonzalez-Vera, E. Hendriksen and O. Njåstad, A Szegö theory for rational functions, Technical Report TW-131, K.U. Leuven, Dept. of Computer Science, May 1990.

7. A. Bultheel, P. Gonzalez-Vera, E. Hendriksen and O. Njåstad, The computation of orthogonal rational functions and their interpolating properties, Numerical Algorithms 2 (1992) 85-114.

8. A. Bultheel, P. Gonzalez-Vera, E. Hendriksen and O. Njåstad, A Favard theorem for orthogonal rational functions on the unit circle, International Congress on Extrapolation and Rational Approxiamtion, Tenerife 1992.

9. A. Bultheel, P. Gonzalez-Vera, E. Hendriksen and O. Njåstad, Moment problems and orthogonal functions, J. Comp. Appl. Math., to appear.

10. A. Bultheel, P. Gonzalez-Vera, E. Hendriksen and O. Njåstad, Orthogonal rational functions with poles on the unit circle, Preprint (1991).

11. T.S. Chihara, Introduction to Orthogonal Polynomials, Mathematics and Its Applications Series, Gordon \& Breach, New York/London/Paris, 1978.

12. P. Delsarte, Y. Genin and Y. Kamp, On the role of the Nevanlinna-Pick problem in circuit and system theory, Int. J. Circuit Th. Appl. 9 (1981) 177-187.

13. P. Dewilde and H. Dym, Schur recursion, error formulas, and convergence of rational estimators for stationary stochastic sequences, IEEE Trans. on Information Theory, IT 27 (1981) 446-461. 
14. M.M. Djrbashian, A survey on the theory of orthogonal systems and some open problems, Orthogonal Polynomials: Theory and Practice (Ed. P. Nevai, Kluwer. Dordrecht/Boston/London 1990) 135-146.

15. Ya. L. Geronimus, Polynomials orthogonal on a circle and their applications, Amer. Math. Soc. Translations 104 (Providence, 1954).

16. E. Hendriksen and $\mathrm{O}$. Njåstad, A Favard theorem for rational functions, J. Math. Anal. Appl. 142 (1989) 508-520.

17. E. Hendriksen and O. Njåstad, Positive multipoint Padé continued fractions, Proc. Edinburgh Math. Soc. 32 (1989) 261-269.

18. E. Hendriksen and H. van Rossum, Orthogonal Laurent polynomials, Indag. Math. (Ser. A) 89 (1986), 17-36.

19. W.B. Jones, O. Njåstad and W.J. Thron, Orthogonal Laurent polynomials and the strong Hamburger moment problem, J. Math. Anal. Appl. 98 (1984) 528-554.

20. W.B. Jones, O. Njåstad, and W.J. Thron, Two-point Padé expansions for a family of analytic functions, J. Comput. Appl. Math. 9 (1983), 105-123.

21. W.B. Jones, O. Njåstad and W.J. Thron, Continued fractions associated with the trigonometric and other strong moment problems, Constr. Approx. 2 (1986) 197-211.

22. W.B. Jones, O. Njåstad and W.J. Thron, Moment theory, orthogonal polynomials. quadrature, and continued fractions associated with the unit circle, Bull. Lond. Math. Soc. 21 (1989) 113-152.

23. W.B. Jones and W.J. Thron, Continued fractions: Analytic theory and applications, Encyclopedia of Mathematics and its Applications, No. 11, Addison-Wesley, Reading, Mass., 1980. (Now distributed by Cambridge Univ. Press.).

24. W.B. Jones and W.J. Thron, Orhogonal Laurent polynomials and Gaussian quadrature, Quantum Mechanics in Mathematics, Chemistry and Physics (Ed. K.E. Gustafson and W.P. Reinhardt, Plenum, New York/London 1981) 449-455.

25. M.G. Krein and A.A. Nudelman, The Markov moment problem and extremal problems, Transl. Math. Monographs, American Mathematical Society No. 50 (Providence 1977).

26. R. Nevanlinna, Über beschränkte Functionen die in gegebenen Punkten vorgeschriebene Werte annehmen, Ann. Acad. Sci. Fenn. Sec. A, 13 (1919) No. 1

27. O. Njåstad, An extended Hamburger moment problem, Proc. Edinburgh Math. Soc. Ser. II, 28 (1985) 167-183. 
28. O. Njåstad, Unique solvability of an extended Hamburger moment problem, J. Math. Anal. Appl. 124 (1987) 502-519.

29. O. Njåstad, Orthogonal rational functions with poles in a finite subset of $R$, Orthogonal Polynomials and their Applications, Springer Lecture Notes in Mathematics 1329 (Ed. Alfaro et al., Berlin 1988) 300-307.

30. O. Njåstad, Multipoint Padé approximation and orthogonal rational functions, Nonlinear Numerical Methods and Rational Approximation (ed.: A. Cuyt, D. Reidel Publ. Comp., Dordrecht 198S) 259-270.

31. O. Njåstad, Solution of the strong Hamburger moment problem by Laurent continued fractions, Appl. Numer. Math. 4 (1988), 351-360.

32. O. Njåstad and W.J. Thron, The theory of sequences of orthogonal $L$-polynomials, in Padé Approximants and Continued Fractions (H. Waadeland and H. Wallin, Det Kongelige Norske Videnskabers Selskab, Skrifter, 1983).

33. G. Pick, Über die Besrchänkungen analytischer Funktionen welche durch vorgegebene Funktionswerte bewirkt werden, Math. Ann. 77 (1916) 7-23.

34. G. Szegö, Orthogonal polynomials (Amer. Math. Soc. Providence, R.I., 1939). 